\title{
THE RECURRENCE RELATIONS OF ORDER STATISTICS MOMENTS FOR POWER LOMAX DISTRIBUTION
}

\author{
DEVENDRA KUMAR ${ }^{\star}$ \\ Department of Statistics, Central University of Haryana, India \\ Email: devendrastats@gmail.com \\ SANKU DEY \\ Department of Statistics, St. Anthony's College, Shillong, Meghalaya, India \\ MAZEN NASSAR \\ Department of Statistics, Faculty of Commerce, Zagazig University, Egypt \\ PREETI YADAV \\ Department of Statistics, Central University of Haryana, India
}

SUMMARY

The power Lomax distribution due to Rady et al. (2016) is an alternative to and provides better fits for bladder cancer data (Lee and Wang, 2003) than the Lomax, exponential Lomax, Weibull Lomax, extended Poisson Lomax and beta Lomax distributions. Exact explicit expressions as well as recurrence relations for the single and double (product) moments have been derived from the power Lomax distribution. These recurrence relations enable computation of the mean, variance, skewness and kurtosis of all order statistics for all sample sizes in a simple and efficient manner. By using these relation, the mean, variance, skewness and kurtosis of order statistics for sample sizes up to 5 for various values of shape and scale parameters are tabulated. Finally, remission times (in months) of bladder cancer patients have been analyzed to show how the proposed relations work in practice

Keywords and phrases: Order statistics, Single and product moments, Recurrence relations

AMS Classification: 62G30, 65C60

\section{Introduction}

Order statistics and its functions are of great significance in reliability theory, the life-length of the $r$-out-of- $n$ system made up of $n$ identical components with independent life-lengths which is the $(n-r+1)$ th order statistic in a sample of size $n, X_{n-r+1: n}$. When $r=1$, it is known as the parallel system. System will function as long as any of the $n$ components survives. However, if $r=n$, it is known as a series system. It has wide applicability in practical problems such as characterization of probability distributions and goodness-of-fit tests,

* Corresponding author

(c) Institute of Statistical Research and Training (ISRT), University of Dhaka, Dhaka 1000, Bangladesh. 
entropy estimation, analysis of censored samples, reliability analysis, quality control and strength of materials; see Arnold et al. (1992), David and Nagaraja (2003), and the references therein for more details.

The usage of moments of order statistics can be especially observed in areas such as quality control testing, reliability theory where a practitioner needs to predict the failure of future items based on the times of a few early failures. These predictions are often based on moments of order statistics. In recent times, the moments of order statistics have been tabulated quite extensively for several distributions (Arnold et al., 1992; David, 1981; Joshi, 1978; Balakrishnan and Joshi, 1982; Balakrishnan and Malik, 1985; Balakrishnan et al., 1988; Malik et al., 1988; Mohie El-Din et al., 1991; Kumar et al., 2016; Kumar and Dey, 2017; and Kumar, 2017). Balakrishnan and Malik (1986) established exact and explicit expressions for the means and product moments of order statistics from the linear-exponential distribution; also they established some recurrence relations for both single and product moments of order statistics for the same model. Several papers dealing with characterization of distribution through properties of order statistics are appeared in the literature see Lin (1988), Kamps (1991) and Mohie El-Din et al. (1991).

The recurrence relations of order statistics and its identities are quite useful in reducing the number of operations necessary to obtain a general form for the function as they reduce the amount of direct computation, time and labor. This feature has been well documented in the statistical literature (Arnold and Balakrishnan, 1989). Besides, they are used in characterizing the distributions, which play in integral role for the identification of population distribution from the properties of the sample. The computation of moments of order statistics is a challenging task for many distributions. For this reason, recursive computational methods are often sought.

Rady et al. (2016) introduced three parameter power Lomax (POLO) distribution and obtained some statistical and reliability properties, and also estimated its parameters by maximum likelihood method. A random variable $X$ has the POLO distribution with parameters $\alpha, \beta$ and $\lambda$ if its cumulative distribution function (cdf) is

$$
F(x ; \alpha, \beta, \lambda)=1-\left(\frac{\lambda}{x^{\beta}+\lambda}\right)^{\alpha}, x>0, \alpha, \beta, \lambda>0
$$

and corresponding probability density function (pdf) is

$$
f(x ; \alpha, \beta, \lambda)=\alpha \beta \lambda^{\alpha} x^{\beta-1}\left(x^{\beta}+\lambda\right)^{-\alpha-1}, x>0, \alpha, \beta, \lambda>0
$$

The hazard function of the POLO distribution is given by

$$
h(x)=\frac{\alpha \beta x^{\beta-1}}{x^{\beta}+\lambda}, x>0, \alpha, \beta, \lambda>0
$$

where $\alpha$ and $\beta$ are the shape parameter and $\lambda$ is the scale parameter of the distribution. Note that Lomax distribution is a member of POLO distribution if $\beta=1$.

In this paper, recurrence relations for all single and product moments of order statistics are derived in a simple recursive manner. The so-obtained relationships enables computation of all the moments of order statistics using some mathematical software (Mathematica, Maple). The rest of the paper is organized as follows. In Section 2, two lemmas are derived for obtaining single moments of order statistics. In Section 3 , single and double moments of order statistics are derived. In Section 4, recurrence relations for the single moments and double moments of order statistics are obtained. Tabulations of mean, variance, skewness and kurtosis of order statistics are given in Section 5. The analysis of one real data set has been presented in Section 6. Some concluding remarks are addressed in Section 7. 


\section{Technical Lemmas}

Two technical lemmas are illustrated below:

Lemma 2.1. Let $F(x)$ and $f(x)$ be given by (1.1) and (1.2), respectively. For $a>0, b>0$ let

$$
I(a, b)=\int_{0}^{\infty} x^{a}[1-F(x)]^{b} f(x) d x
$$

then

$$
I(a, b)=\alpha \lambda^{\frac{a}{\beta}} \frac{\Gamma\left(\frac{a+\beta}{\beta}\right) \Gamma\left(1+\alpha(b+1)-\frac{a+\beta}{\beta}\right)}{\Gamma(\alpha(b+1)+1)} .
$$

Proof. We can write

$$
\begin{aligned}
I(a, b) & =\int_{0}^{\infty} x^{a}[1-F(x)]^{b} f(x) d x \\
& =\int_{0}^{\infty} x^{a}\left[\frac{\lambda}{\left(x^{\beta}+\lambda\right)}\right]^{\alpha b} \alpha \beta \lambda^{\alpha} x^{\beta-1}\left(x^{\beta}+\lambda\right)^{-\alpha-1} d x \\
& =\alpha \beta \lambda^{\alpha(b+1)} \int_{0}^{\infty} x^{a+\beta-1}\left(x^{\beta}+\lambda\right)^{-\alpha(b+1)-1} d x .
\end{aligned}
$$

The result follows by using equation (3.241) in Gradshteyn and Ryzhik (2014) to calculate the integral in (2.1). The proof is complete.

Lemma 2.2. Let $F(x)$ and $f(x)$ be given by (1.1) and (1.2), respectively. For $a>0, b>0, p>0$ and $q>0$, let

$$
K(p, q, a, b)=\int_{0}^{\infty} \int_{x}^{\infty} x^{p} y^{q}[1-F(x)]^{a}[1-F(y)]^{b} f(x) f(y) d y d x
$$

then

$$
K(p, q, a, b)=\alpha^{2} \lambda^{\frac{q+p}{\beta}} \sum_{i=0}^{\frac{q}{\beta}}\left(\begin{array}{c}
\frac{q}{\beta} \\
i
\end{array}\right) \frac{(-1)^{i}}{c \beta} \times \frac{\Gamma\left(\frac{p+\beta}{\beta}\right) \Gamma\left(1+\alpha(a+c+1)-\frac{p+\beta}{\beta}\right)}{\Gamma(\alpha(a+c+1)+1)},
$$

where $c=(1 / \beta)[\alpha(b+1)+i-(q / \beta)]$,

Proof. Here

$$
\begin{aligned}
K(p, q, a, b) & =\int_{0}^{\infty} \int_{x}^{\infty} x^{p} y^{q}[1-F(x)]^{a}[1-F(y)]^{b} f(x) f(y) d y d x \\
& =\int_{0}^{\infty} x^{p}[1-F(x)]^{a} f(x) I(x) d x
\end{aligned}
$$

where

$$
I(x)=\int_{x}^{\infty} y^{q}[1-F(y)]^{b} f(y) d y=\alpha \int_{0}^{[\bar{F}(x)]^{\frac{1}{\beta}}} \lambda^{\frac{q}{\beta}}(1-z)^{\frac{q}{\beta}} z^{\alpha(b+1)-1-\frac{q}{\beta}} d z .
$$


By using binomial expansion, the following expression can be obtained:

$$
I(x)=\alpha \lambda^{\frac{q}{\beta}} \sum_{i=0}^{\frac{q}{\beta}}\left(\begin{array}{c}
\frac{q}{\beta} \\
i
\end{array}\right)(-1)^{i} \int_{0}^{[\bar{F}(x)]^{\frac{1}{\beta}}} z^{c \beta} d z=\alpha \lambda^{\frac{q}{\beta}} \sum_{i=0}^{\frac{q}{\beta}}\left(\begin{array}{c}
\frac{q}{\beta} \\
i
\end{array}\right) \frac{(-1)^{i}}{c \beta}[\bar{F}(x)]^{c} .
$$

Hence,

$$
K(p, q, a, b)=\alpha \lambda^{\frac{q}{\beta}} \sum_{i=0}^{\frac{q}{\beta}}\left(\begin{array}{c}
\frac{q}{\beta} \\
i
\end{array}\right) \frac{(-1)^{i}}{c \beta} \int_{0}^{\infty} x^{p}[1-F(x)]^{(a+c)} f(x) d x .
$$

The result follows by using lemma (2.2) to calculate the integral in (2.2). The proof is complete.

\section{Moment of Order Statistics}

In this section, the exact explicit forms for the single and double moments of order statistics from the POLO distribution are derived.

\subsection{Single Moments}

The single moments of order statistics are very important to calculate the variance and draw the inferential techniques for the underlying distribution. In the following, the single moments of order statistics from the POLO distribution are derived. Let $X_{(1)} \leq \cdots \leq X_{(n)}$ denote the order statistics corresponding to $X_{1}, \ldots, X_{n}$ from the POLO distribution given in Equation (2) with its cdf in Equation (1). Then pdf of the $r$ th order statistic is

$$
f_{X_{(r)}}(x)=C_{r: n}[F(x)]^{r-1}[1-F(x)]^{n-r} f(x), x>0, r=1, \ldots, n,
$$

where

$$
C_{r: n}=\frac{n !}{(r-1) !(n-r) !}
$$

Using binomial expansion, (3.1) can be rewritten as

$$
f_{X_{(r)}}(x)=C_{r: n} \sum_{\ell=0}^{r-1}(-1)^{\ell}[1-F(x)]^{\ell+n-r} f(x) d x .
$$

The $j$ th moment of the $r$ th-order statistic $\mu_{r: n}^{(j)}=E\left(X_{r}^{(j)}\right)$ is given by

$$
\mu_{r: n}^{(j)}=C_{r: n} \sum_{\ell=0}^{r-1}\left(\begin{array}{c}
r-1 \\
\ell
\end{array}\right)(-1)^{\ell} \int_{0}^{\infty} x^{j}[1-F(x)]^{n-r+\ell} f(x) d x
$$

Using (3.2), Lemma 2.1, the moments of the $r$ th order statistic can be written as:

$$
\mu_{r: n}^{(j)}=C_{r: n} \sum_{\ell=0}^{r-1}\left(\begin{array}{c}
r-1 \\
\ell
\end{array}\right)(-1)^{\ell} I(j, n-r+\ell)
$$


The validity of the single moments of order statistics in Equation (3.3) can be checked by using Arnold et al. (1992)

$$
\sum_{r=1}^{n} \mu_{r: n}=n E(X) .
$$

In particular, the mean order statistic and the variance of order statistic are

$$
\begin{aligned}
\mu_{r: n}^{(1)} & =C_{r: n} \sum_{\ell=0}^{r-1}\left(\begin{array}{c}
r-1 \\
\ell
\end{array}\right)(-1)^{\ell} I(1, n-r+\ell) \\
& =\alpha \lambda^{\frac{1}{\beta}} C_{r: n} \sum_{\ell=0}^{r-1}\left(\begin{array}{c}
r-1 \\
\ell
\end{array}\right) \frac{(-1)^{\ell} \Gamma\left(\frac{1+\beta}{\beta}\right) \Gamma\left(1+\alpha(n-r+\ell+1)-\frac{1+\beta}{\beta}\right)}{\Gamma(\alpha(n-r+\ell+1)+1)} \\
\sigma_{r: n}^{(2)} & =\mu_{r: n}^{(2)}-\left[\begin{array}{c}
\mu_{r: n}^{(1)} \\
{ }^{2}
\end{array}\right. \\
& =\alpha \lambda^{\frac{2}{\beta}} C_{r: n} \sum_{\ell=0}^{r-1}\left(\begin{array}{c}
r-1 \\
\ell
\end{array}\right) \frac{(-1)^{\ell} \Gamma\left(\frac{2+\beta}{\beta}\right) \Gamma\left(1+\alpha(n-r+\ell+1)-\frac{2+\beta}{\beta}\right)}{\Gamma(\alpha(n-r+\ell+1)+1)}-\left[\mu_{r: n}^{(1)}\right]^{2} .
\end{aligned}
$$

Remark 1. If $\beta=1$ in (3.3), the explicit expression of order statistics for Lomax distribution can be obtained.

\subsection{Double Moments}

The double moments of order statistics are very important to calculate the variance and draw the inferential techniques for the underlying distribution. In the following, the double moments of order statistics from the POLO distribution are derived.

Let $X_{(1)} \leq \cdots \leq X_{(n)}$ denote the order statistics corresponding to $X_{1}, \ldots, X_{n}$ from the POLO distribution given in (1.2) with its cdf in (1.1). The joint pdf of the $r$ th and $s$ th order statistics is

$$
f_{X_{(r)}, X_{(s)}}(x, y)=C_{r, s: n}[F(x)]^{r-1}[F(y)-F(x)]^{s-1-r}[1-F(y)]^{n-s} f(x) f(y)
$$

for $r, s=1,2 \ldots, n, r<s, 0<x<y$, where

$$
C_{r, s: n}=\frac{n !}{(r-1) !(s-r-1) !(n-s) !} .
$$

Using binomial expansion,(3.4) can be rewritten as

$$
\begin{aligned}
f_{X_{(r)}, X_{(s)}}(x, y)= & C_{r, s: n} \sum_{\ell_{1}=0}^{r-1} \sum_{\ell_{2}=0}^{s-r-1}\left(\begin{array}{c}
r-1 \\
\ell_{1}
\end{array}\right)\left(\begin{array}{c}
s-r-1 \\
\ell_{2}
\end{array}\right)(-1)^{\ell_{1}+\ell_{2}} \\
& \times \int_{0}^{\infty} \int_{x}^{\infty} x^{p} y^{q}[1-F(x)]^{s-r-1-\ell_{1}+\ell_{2}}[1-F(y)]^{n-s+\ell_{1}} f(x) f(y) d y d x,
\end{aligned}
$$

for $0<x<y$. Then, the double (product) moments of order statistics

$$
\mu_{r, s: n}^{(p, q)}=E\left(X_{r: n}^{(p)} X_{s: n}^{(q)}\right)
$$

is given by

$$
\begin{aligned}
\mu_{r, s: n}^{(p, q)}= & C_{r, s: n} \sum_{\ell_{1}=0}^{r-1} \sum_{\ell_{2}=0}^{s-r-1}\left(\begin{array}{c}
r-1 \\
\ell_{1}
\end{array}\right)\left(\begin{array}{c}
s-r-1 \\
\ell_{2}
\end{array}\right)(-1)^{\ell_{1}+\ell_{2}} \\
& \times K\left(p, q,\left(s-r-1-\ell_{1}+\ell_{2}\right),\left(n-s+\ell_{1}\right)\right) .
\end{aligned}
$$


The validity of the double moments of order statistics in Equation (3.5) can be checked by using Arnold et al. (1992)

$$
\sum_{r=1}^{n-1} \sum_{s=r+1}^{n} \mu_{r, s: n}=\left(\begin{array}{l}
n \\
2
\end{array}\right)[E(X)]^{2}
$$

In particular, the covariance of order statistics is

$$
\begin{aligned}
\mu_{r, s: n}^{(1,1)}= & C_{r, s: n} \sum_{\ell_{1}=0}^{r-1} \sum_{\ell_{2}=0}^{s-r-1}\left(\begin{array}{c}
r-1 \\
\ell_{1}
\end{array}\right)\left(\begin{array}{c}
s-r-1 \\
\ell_{2}
\end{array}\right)(-1)^{\ell_{1}+\ell_{2}} \\
& \times K\left(1,1,\left(s-r-1-\ell_{1}+\ell_{2}\right),\left(n-s+\ell_{1}\right)\right) \\
= & \alpha^{2} \lambda^{\frac{2}{\beta}} C_{r, s: n} \sum_{\ell_{1}=0}^{r-1} \sum_{\ell_{2}=0}^{s-r-1} \sum_{i=0}^{\frac{1}{\beta}}\left(\begin{array}{c}
r-1 \\
\ell_{1}
\end{array}\right)\left(\begin{array}{c}
s-r-1 \\
\ell_{2}
\end{array}\right) \frac{(-1)^{\ell_{1}+\ell_{2}+i}}{\left(\alpha\left(n-s+\ell_{1}+1\right)+i-\frac{1}{\beta}\right)} \\
& \times \frac{\Gamma\left(\frac{1+\beta}{\beta}\right) \Gamma\left(1+\alpha\left(s-r-\ell_{1}+\ell_{2}+\frac{1}{\beta}\left(\alpha\left(n-s+\ell_{1}+1\right)+i-\frac{1}{\beta}\right)\right)-\frac{1+\beta}{\beta}\right)}{\Gamma\left(\alpha\left(s-r-\ell_{1}+\ell_{2}+\frac{1}{\beta}\left(\alpha\left(n-s+\ell_{1}+1\right)+i-\frac{1}{\beta}\right)\right)+1\right)} .
\end{aligned}
$$

Remark 2. Put $\beta=1$ in (3.5), the explicit expression of product moment of order statistics for Lomax distribution can be obtained.

\section{Recurrence Relations of Order Statistics}

Here, the recurrence relation for the single and double moments of order statistics from the POLO distribution are derived.

\subsection{Recurrence Relation for Single Moments}

Theorem 1. For the distribution given in (1.2) and for $1 \leq r \leq n$ and $j=1,2, \ldots$ then

$$
\mu_{r: n}^{(j)}=\frac{\alpha \beta(n-r+1)}{\lambda(j+\beta)}\left(\mu_{r: n}^{(j+\beta)}-\mu_{r-1: n}^{(j+\beta)}\right)-\frac{1}{\lambda} \mu_{r: n}^{(j+\beta)} .
$$

Proof. Clearly (1.1) and (1.2) gives

$$
\left(\frac{x^{\beta}}{\lambda}+1\right) f(x)=\frac{\alpha \beta x^{\beta-1}}{\lambda} \bar{F}(x) .
$$

Therefore, for $j=1,2, \ldots$

$$
\begin{aligned}
\mu_{r: n}^{(j)}+\frac{1}{\lambda} \mu_{r: n}^{(j+\beta)} & =C_{r: n} \int_{0}^{\infty}\left(x^{j}+\frac{x^{j+\beta}}{\lambda}\right)[F(x)]^{r-1}[1-F(x)]^{n-r} f(x) d x \\
& =C_{r: n} \int_{0}^{\infty} x^{j}\left(1+\frac{x^{\beta}}{\lambda}\right)[F(x)]^{r-1}[1-F(x)]^{n-r} f(x) d x \\
& =C_{r: n} \frac{\alpha \beta}{\lambda} \int_{0}^{\infty} x^{j+\beta-1}[F(x)]^{r-1}[1-F(x)]^{n-r+1} d x .
\end{aligned}
$$


Integrating by parts, the above expression can be written as

$$
\begin{aligned}
\mu_{r: n}^{(j)}+\frac{1}{\lambda} \mu_{r: n}^{(j+\beta)}= & C_{r: n} \frac{\alpha \beta}{\lambda}\left[\frac{n-r+1}{j+\beta} \int_{0}^{\infty} x^{j+\beta}[F(x)]^{r-1}[1-F(x)]^{n-r} f(x) d x\right. \\
& \left.-\frac{r-1}{j+\beta} \int_{0}^{\infty} x^{j+\beta}[F(x)]^{r-2}[1-F(x)]^{n-r+1} f(x) d x\right] \\
= & \frac{\alpha \beta}{\lambda}\left[\frac{n-r+1}{j+\beta} C_{r: n} \int_{0}^{\infty} x^{j+\beta}[F(x)]^{r-1}[1-F(x)]^{n-r} f(x) d x\right. \\
& \left.-\frac{r-1}{j+i \beta} C_{r: n} \int_{0}^{\infty} x^{j+\beta}[F(x)]^{r-2}[1-F(x)]^{n-r+1} f(x) d x\right] \\
= & \frac{\alpha \beta}{\lambda}\left[\left(\frac{n-r+1}{j+\beta}\right) \mu_{r: n}^{(j+\beta)}-\left(\frac{r-1}{j+\beta}\right) \frac{C_{r: n}}{C_{r-1: n}} \mu_{r-1: n}^{(j+\beta)}\right] .
\end{aligned}
$$

The result follows.

In particular, upon setting $r=1$ in Theorem 1 , the following result can be deduced.

Corollary 4.1. For the POLO distribution given in (1.2),

$$
\mu_{1: n}^{(j)}=\frac{1}{\lambda} \mu_{1: n}^{(j+\beta)}\left(\frac{\alpha \beta n}{j+\beta}-1\right)
$$

\subsection{Recurrence Relation for Double Moments}

Theorem 2. For the distribution given in (1.2) and for $1 \leq r<s \leq n$ and $i, j=1,2, \ldots$

$$
\mu_{r, s: n}^{(i, j)}=\alpha^{2} \beta^{2} \sum_{i_{1}=0}^{\infty} \sum_{i_{2}=0}^{\infty} \frac{(-1)^{i_{1}+i_{2}-2}}{\lambda^{\left(i_{1}+i_{2}\right)}}\left[\frac{n-s+1}{j+i_{2} \beta}\left(I_{1}-I_{2}\right)-\frac{s-r-1}{j+i_{2} \beta}\left(I_{3}-I_{4}\right)\right],
$$

where

$$
\begin{aligned}
& I_{1}=-\frac{n}{i+i_{1} \beta} \mu_{r-1, s-1 ; n-1}^{\left(i+i_{1} \beta, j+i_{2} \beta\right)}+\frac{n}{i+i_{1} \beta} \mu_{r, s-1 ; n-1}^{\left(i+i_{1} \beta, j+i_{2} \beta\right)}, \\
& I_{2}=-\frac{r}{i+i_{1} \beta} \mu_{r, s ; n}^{\left(i+i_{1} \beta, j+i_{2} \beta\right)}+\frac{n r}{i+i_{1} \beta} \mu_{r+1, s ; n}^{\left(i+i_{1} \beta, j+i_{2} \beta\right)}, \\
& I_{3}=-\frac{n}{(s-r-1)\left(i+i_{1} \beta\right)} \mu_{r-1, s-2 ; n-1}^{\left(i+i_{1} \beta, j+i_{2} \beta\right)}+\frac{n(n-s+1)}{(s-r-2)\left(i+i_{1} \beta\right)} \mu_{r, s-2 ; n-1}^{\left(i+i_{1} \beta, j+i_{2} \beta\right)}, \\
& I_{4}=-\frac{r(n-s+1)}{(s-r-1)\left(i+i_{1} \beta\right)} \mu_{r, s-1 ; n}^{\left(i+i_{1} \beta, j+i_{2} \beta\right)}+\frac{r(n-s+1)}{(s-r-1)\left(i+i_{1} \beta\right)} \mu_{r+1, s-1 ; n}^{\left(i+i_{1} \beta, j+i_{2} \beta\right)} .
\end{aligned}
$$

Proof. Clearly (1.1) and (1.2) gives

$$
f(x)=\alpha \beta \sum_{i=0}^{\infty} \frac{(-1)^{i-1} x^{\beta i-1} \bar{F}(x)}{\lambda^{i}} .
$$


Therefore, for $i, j=1,2, \ldots$

$$
\begin{aligned}
\mu_{r, s: n}^{(i, j)}= & \frac{\alpha^{2} \beta^{2} C_{r, s: n}}{\lambda^{2}} \int_{0}^{\infty} \int_{x}^{\infty} \frac{x^{i+\beta-1} y^{j+\beta-1}}{\left(1+\frac{x^{\beta}}{\lambda}\right)\left(1+\frac{y^{\beta}}{\lambda}\right)} F_{x, y}^{\star}(r-1, s-r-1, n-s+1,1) d y d x \\
= & \alpha^{2} \beta^{2} C_{r, s: n} \sum_{i_{1}=0}^{\infty} \sum_{i_{2}=0}^{\infty} \frac{(-1)^{i_{1}+i_{2}-2}}{\lambda^{\left(i_{1}+i_{2}\right)}} \int_{0}^{\infty} \int_{x}^{\infty} x^{i+i_{1} \beta-1} y^{j+i_{2} \beta-1} \\
& \quad \times F_{x, y}^{\star}(r-1, s-r-1, n-s+1,1) d y d x \\
= & \alpha^{2} \beta^{2} C_{r, s: n} \sum_{i_{1}=0}^{\infty} \sum_{i_{2}=0}^{\infty} \frac{(-1)^{i_{1}+i_{2}-2}}{\lambda^{\left(i_{1}+i_{2}\right)}} \int_{0}^{\infty} \int_{x}^{\infty} x^{i+i_{1} \beta-1} y^{j+i_{2} \beta-1} \\
& \times\left[F_{x, y}^{\star}(r-1, s-r-1, n-s+1,1)-F_{x, y}^{\star}(r, s-r-1, n-s+1,1)\right] d y d x
\end{aligned}
$$

where

$$
F_{x, y}^{\star}(k, l, m, r)=[F(x)]^{k}[F(y)-F(x)]^{l}[1-F(y)]^{m}[1-F(x)]^{r} .
$$

Integrating by parts with respect to $y$, the following expression can be obtained

$$
\mu_{r, s: n}^{(i, j)}=\sum_{i_{1}=0}^{\infty} \sum_{i_{2}=0}^{\infty} \frac{\alpha^{2} \beta^{2}(-1)^{i_{1}+i_{2}-2} C_{r, s: n}}{\lambda^{\left(i_{1}+i_{2}\right)}}\left[\frac{(n-s+1)\left(I_{1}-I_{2}\right)}{\left(j+i_{2} \beta\right)}-\frac{(s-r-1)\left(I_{3}-I_{4}\right)}{\left(j+i_{2} \beta\right)}\right]
$$

where

$$
\begin{aligned}
& I_{1}=\int_{0}^{\infty} \int_{x}^{\infty} x^{i+i_{1} \beta-1} y^{j+i_{2} \beta} F_{x, y}^{\star}(r-1, s-r-1, n-s, 0) d y d x \\
& I_{2}=\int_{0}^{\infty} \int_{x}^{\infty} x^{i+i_{1} \beta-1} y^{j+i_{2} \beta} F_{x, y}^{\star}(r, s-r-1, n-s, 0) d y d x \\
& I_{3}=\int_{0}^{\infty} \int_{x}^{\infty} x^{i+i_{1} \beta-1} y^{j+i_{2} \beta} F_{x, y}^{\star}(r-1, s-r-2, n-s+1,0) d y d x \\
& I_{4}=\int_{0}^{\infty} \int_{x}^{\infty} x^{i+i_{1} \beta-1} y^{j+i_{2} \beta} F_{x, y}^{\star}(r, s-r-2, n-s+1,0) d y d x .
\end{aligned}
$$

Integrating by parts with respect to $x$, the following results can be obtained

$$
\begin{aligned}
& I_{1}=-\frac{(r-1) J_{1}}{i+i_{1} \beta}+\frac{(s-r-1) J_{2}}{i+i_{1} \beta}, \quad I_{2}=-\frac{r J_{3}}{i+i_{1} \beta}+\frac{(s-r-1) J_{4}}{i+i_{1} \beta} \\
& I_{3}=-\frac{(r-1) J_{5}}{i+i_{1} \beta}+\frac{(s-r-2) J_{6}}{i+i_{1} \beta}, \quad I_{4}=-\frac{r J_{7}}{i+i_{1} \beta}+\frac{(s-r-2) J_{8}}{i+i_{1} \beta}
\end{aligned}
$$


where

$$
\begin{aligned}
& J_{1}=\int_{0}^{\infty} \int_{x}^{\infty} \frac{x^{i+i_{1} \beta} F_{x, y}^{\star}(r-2, s-r+1, n-s, 0)}{y^{-\left(j+i_{2} \beta\right)}} d F(x) d F(y)=\frac{\mu_{r-1, s-1 ; n-1}^{\left(i+i_{1} \beta, j+i_{2} \beta\right)}}{C_{r-1, s-1: n-1}} \\
& J_{2}=\int_{0}^{\infty} \int_{x}^{\infty} \frac{x^{i+i_{1} \beta} F_{x, y}^{\star}(r-1, s-r-2, n-s, 0)}{y^{-\left(j+i_{2} \beta\right)}} d F(x) d F(y)=\frac{\mu_{r, s-1 ; n-1}^{\left(i+i_{1} \beta, j+i_{2} \beta\right)}}{C_{r, s-1: n-1}} \\
& J_{3}=\int_{0}^{\infty} \int_{x}^{\infty} \frac{x^{i+i_{1} \beta} F_{x, y}^{\star}(r-1, s-r-1, n-s, 0)}{y^{-\left(j+i_{2} \beta\right)}} d F(x) d F(y)=\frac{\mu_{r, s ; n}^{\left(i+i_{1} \beta, j+i_{2} \beta\right)}}{C_{r, s: n}} \\
& J_{4}=\int_{0}^{\infty} \int_{x}^{\infty} \frac{x^{i+i_{1} \beta} F_{x, y}^{\star}(r-1, s-r-2, n-s, 0)}{y^{-\left(j+i_{2} \beta\right)}} d F(x) d F(y)=\frac{\mu_{r+1, s ; n}^{\left(i+i_{1} \beta, j+i_{2} \beta\right)}}{C_{r+1, s: n-1}} \\
& J_{5}=\int_{0}^{\infty} \int_{x}^{\infty} \frac{x^{i+i_{1} \beta} F_{x, y}^{\star}(r-2, s-r-2, n-s+1,0)}{y^{-\left(j+i_{2} \beta\right)}} d F(x) d F(y)=\frac{\mu_{r-1, s-2 ; n-1}^{\left(i+i_{1} \beta, j+i_{2} \beta\right)}}{C_{r-1, s-2: n-1}} \\
& J_{6}=\int_{0}^{\infty} \int_{x}^{\infty} \frac{x^{i+i_{1} \beta} F_{x, y}^{\star}(r-1, s-r-3, n-s+1,0)}{\left.y^{-\left(j+i_{2} \beta\right.}\right)} d F(x) d F(y)=\frac{\mu_{r, s-2 ; n-1}^{\left(i+i_{1} \beta, j+i_{2} \beta\right)}}{C_{r, s-2, n-1}} \\
& J_{7}=\int_{0}^{\infty} \int_{x}^{\infty} \frac{x^{i+i_{1} \beta} F_{x, y}^{\star}(r-1, s-r-2, n-s+1,0)}{\left.y^{-\left(j+i_{2} \beta\right.}\right)} d F(x) d F(y)=\frac{\mu_{r, s-1 ; n}^{\left(i+i_{1} \beta, j+i_{2} \beta\right)}}{C_{r, s-1: n}} \\
& J_{8}=\int_{0}^{\infty} \int_{x}^{\infty} \frac{x^{i+i_{1} \beta} F_{x, y}^{\star}(r-1, s-r-3, n-s+1,0)}{\left.y_{r+i_{2} \beta}\right)} d F(x) d F(y)=\frac{\mu_{r+1, s-1 ; n}^{\left(i+i_{1} \beta, j+i_{2} \beta\right)}}{C_{r+1, s-1: n}}
\end{aligned}
$$

The result follows by combining (10), (11) - (14) and (15) - (22).

In particular, upon setting $s=r+1$ in Theorem 2 , the following result can be deduced.

Corollary 4.2. For the POLO distribution given in (1.2) and for $1 \leq r \leq n$

$$
\mu_{r, r+1: n}^{(i, j)}=\alpha^{2} \beta^{2} \sum_{i_{1}=0}^{\infty} \sum_{i_{2}=0}^{\infty}(-1)^{i_{1}+i_{2}-2} \lambda^{-\left(i_{1}+i_{2}\right)}\left(\frac{n-r}{j+i_{2} \beta} I_{1}-\frac{n-r}{j+i_{2} \beta} I_{2}\right),
$$

where

$$
\begin{aligned}
& I_{1}=-\frac{n}{i+i_{1} \beta} \mu_{r-1, r ; n-1}^{\left(i+i_{1} \beta, j+i_{2} \beta\right)}+\frac{n}{i+i_{1} \beta} \mu_{r, r ; n-1}^{\left(i+i_{1} \beta, j+i_{2} \beta\right)} \\
& I_{2}=-\frac{r}{i+i_{1} \beta} \mu_{r, r+1 ; n}^{\left(i+i_{1} \beta, j+i_{2} \beta\right)}+\frac{n r}{i+i_{1} \beta} \mu_{r+1, r+1 ; n}^{\left(i+i_{1} \beta, j+i_{2} \beta\right)}
\end{aligned}
$$

\section{Tabulations of Mean, Variance, Skewness and Kurtosis}

The recurrence relations obtained in the preceding sections allow us to evaluate the mean, variance, skewness and kurtosis of all order statistics for all sample sizes in a simple recursive manner. In Tables 2 and 3, the mean values for $\alpha=0.5(0.5) 3$ and $\beta=5$ and 10 and $\lambda=1$ and 2 are reported. Tables 2 and 3 show that the mean of order statistics decreases as $\alpha$ increases. In Tables 4 and 5, the variances of order statistics for different values of $r, s$ and $n$ for $\alpha=0.5(0.5) 3$ and $\beta=5$ and 10 and $\lambda=1$ and 2 are computed. From Tables 4 and 5, one can observe that as $\alpha$ increases, variances of order statistics decreases. Similar conclusion can be drawn for skewness and kurtosis displayed in Tables 6-9, except some cases. Tabular values of mean, variance, skewness and kurtosis are presented in Appendix. Figures 1-4 present the mean, variance, skewness and kurtosis of the first and the last order statistics for $n=3,4$ and 5 for different values of $\alpha$ and $\beta=5$ and 
$\lambda=1$. From these Figures, one can observe that the mean, variance and skewness of the first and the last order statistics decreases as $\alpha$ increases, except the kurtosis of the last order statistics which increase then decrease as $\alpha$ increases.
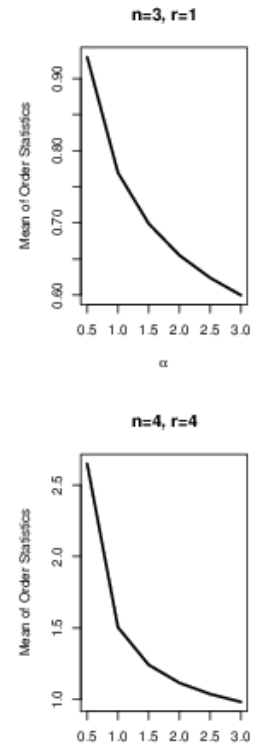

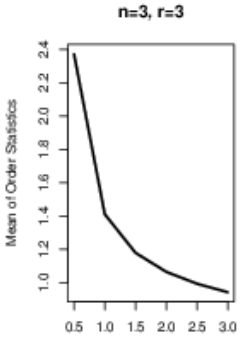

$\alpha$

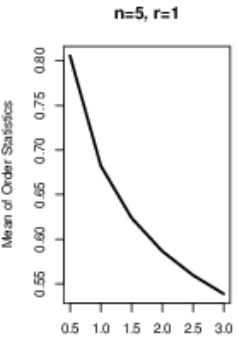

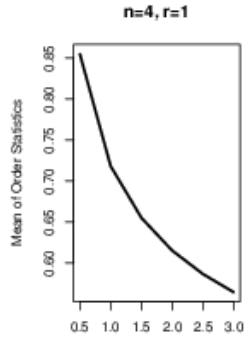

$\alpha$

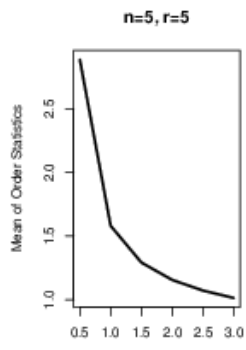

Figure 1: Mean of order statistics for $\beta=5, \lambda=1$ and different values of $\alpha$.

\section{Data Analysis}

In this section, we analyse a real data set corresponding to remission times (in months) of a random sample of 128 bladder cancer patients given in Lee and Wang (2003). Rady et al. (2016) showed that the POLO distribution provides a better fit to this data than Lomax, MCLomax , BLomax and KW Lomax by Lemonte and Cordeiro (2013), exponential Lomax (El-Bassiouny et al., 2015), gamma Lomax (Cordeiro et al., 2013), transmuted exponentiated Lomax (Ashour and Eltehiwy, 2013), Weibull Lomax (Tahir et al., 2015), extended Poisson Lomax (Al-Zahrani, 2015) and exponentiated Lomax (Abdul-Moniem, 2012). They obtained the MLEs of the unknown parameters as: $\hat{\alpha}=2.070, \hat{\beta}=1.428$ and $\hat{\lambda}=34.863$.

The above estimates can be used to know how the minimum and maximum remission times (in months) occur on average in every $n$ patients. These remission times can be estimated by $\mu_{1: n}^{(1)}$ and $\mu_{n: n}^{(1)}$, respectively. Table 1 displays the ML predictions of $\mu_{1: n}^{(1)}$ and $\mu_{n: n}^{(1)}$ for $n=20(20) 140$. Also, the values of variance, skewness and kurtosis for these predictions are presented in Table 1. From Table 1, it is to be noted that the values of $\mu_{1: n}^{(1)}$ decreases when the sample size increases while the corresponding variance decreases as the sample size increases. On the other hand, the values of $\mu_{n: n}^{(1)}$ and the corresponding variance increase as the sample size increase, while the corresponding skewness and kurtosis decreases with the increasing in the sample 

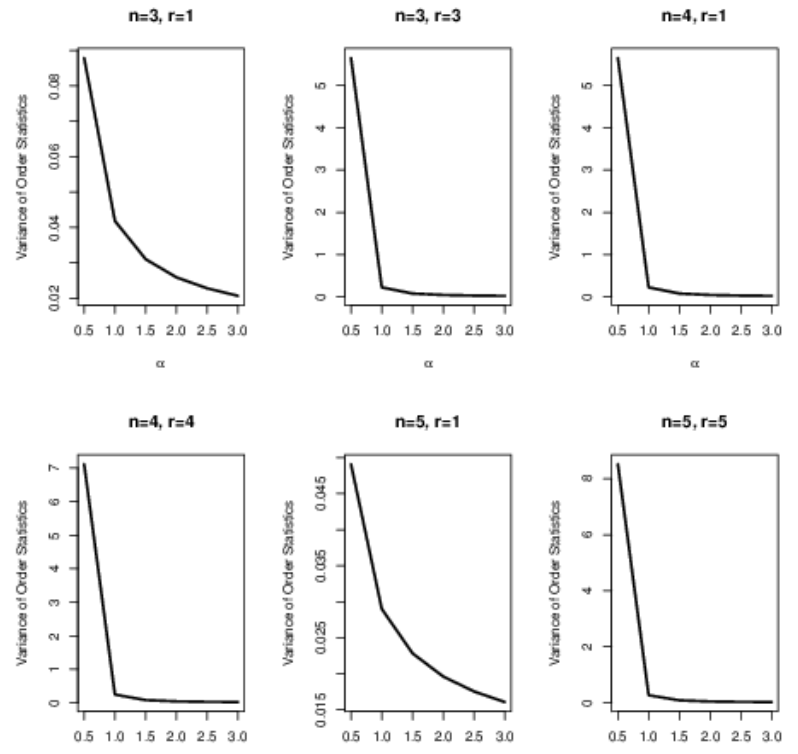

$\alpha$

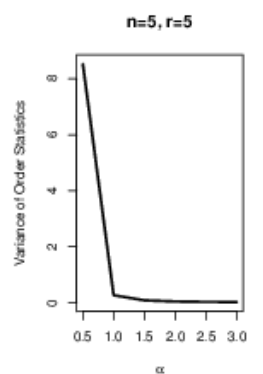

Figure 2: Variance of order statistics for $\beta=5, \lambda=1$ and different values of $\alpha$.
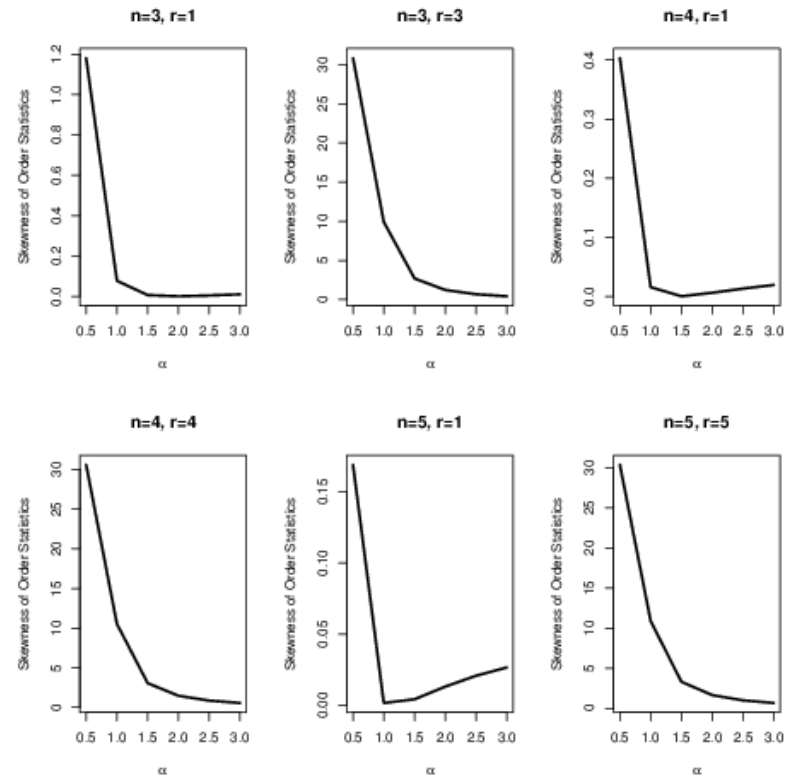

Figure 3: Skewness of order statistics for $\beta=5, \lambda=1$ and different values of $\alpha$. 


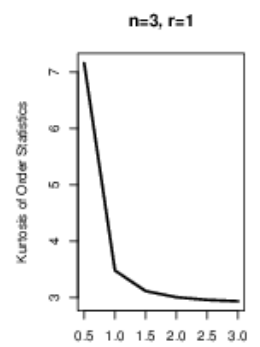

$\alpha$

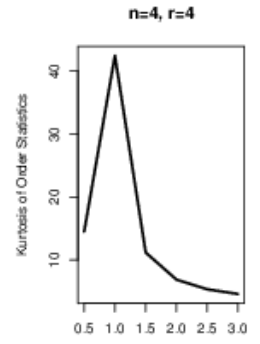

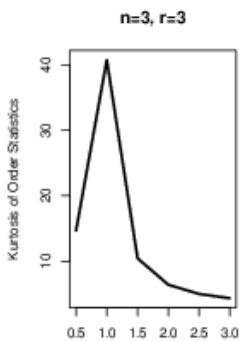

a

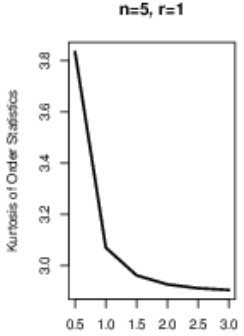

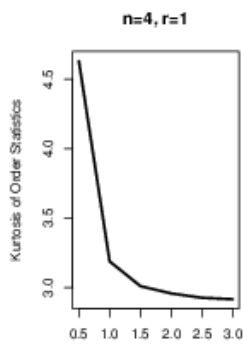

$\alpha$

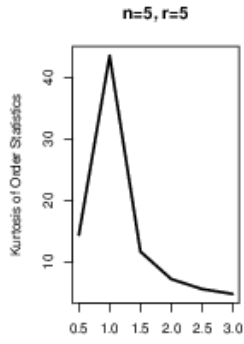

Figure 4: Kurtosis of order statistics for $\beta=5, \lambda=1$ and different values of $\alpha$.

size. Finally, from Table 1, it can be concluded that the minimum expected remission time is 0.21 months and maximum remission time is 83.49 months, respectively.

\section{Conclusion}

In this paper, recurrence relations for single and product moments of order statistics from POLO distribution have been derived. The recurrence relations for moments of order statistics are important because they can be helpful in reducing the amount of direct calculations needed to calculate the moments, and they can be used in a simple recursive manner to express the unknown higher order moments in terms of order statistics thus making the evaluation of higher moments easy. Also they can be used to characterize the distributions. The work is in progress on inferential issues and it will be reported later.

\section{Acknowledgements}

The authors would like to thank the the editor and the referee for their comments which helped improve the paper. 
Table 1: Estimates of $\mu_{1: n}^{(1)}$ and $\mu_{n: n}^{(1)}$ and the corresponding variance, skewness and kurtosis for real data

\begin{tabular}{|c|c|c|c|c|c|c|c|c|}
\hline \multirow[b]{2}{*}{$n$} & \multicolumn{4}{|c|}{$\mu_{1: n}^{(1)}$} & \multicolumn{4}{|c|}{$\mu_{n: n}^{(1)}$} \\
\hline & MLE & Variance & Skewness & Kurtosis & MLE & Variance & Skewness & Kurtosis \\
\hline 20 & 0.817 & 0.350 & 1.245 & 5.111 & 40.178 & 1039.429 & 2.948 & 22.334 \\
\hline 40 & 0.499 & 0.128 & 0.062 & 0.458 & 52.581 & 1643.598 & 2.327 & 14.512 \\
\hline 60 & 0.375 & 0.072 & 0.317 & 0.205 & 61.237 & 2151.711 & 1.986 & 11.014 \\
\hline 80 & 0.306 & 0.048 & 1.330 & 1.440 & 68.110 & 2606.208 & 1.755 & 8.948 \\
\hline 100 & 0.262 & 0.035 & 2.733 & 4.116 & 73.906 & 3024.651 & 1.583 & 7.558 \\
\hline 120 & 0.230 & 0.027 & 4.050 & 7.717 & 78.967 & 3416.468 & 1.448 & 6.549 \\
\hline 140 & 0.207 & 0.022 & 4.974 & 11.364 & 83.490 & 3787.462 & 1.338 & 5.778 \\
\hline
\end{tabular}

\section{References}

Abdul-Moniem, I. B. (2012), "Recurrence relations for moments of lower generalized order statistics from exponentiated Lomax distribution and its characterization”, Int. J. Math. Arch, 3, 2144-2150.

Al-Zahrani, B. (2015), “An extended Poisson-Lomax distribution”, Adv. Math. Sci J., 4, 79-89.

Arnold, B. C. and Balakrishnan, N. (1989), Relations, Bounds, and Approximations for Order Statistics, Lecture Notes in Statistics, 53, Springer-Verlag, New York.

Arnold, B. C., Balakrishnan, N. and Nagaraja, H. N. (1992), A First Course in Order Statistics, John Wiley, New York.

Ashour, S. and Eltehiwy, M. (2013), "Transmuted exponentiated Lomax distribution”, Aust. J. Basic Appl. Sci., 7(7), 658-667.

Balakrishnan, N. and Joshi, P. C. (1982), "Moments of order statistics from doubly truncated Pareto distribution", J. Indian Statist. Assoc., 20, 109-117.

Balakrishnan, N., Malik, H. J., and Ahmad, S. E. (1988), "Recurrence relations and identities for moments of order statistics, II: Specific continuous distributions", Communications in Statistics - Theory and Methods, 17, 2657-2694.

Balakrishnan, N. and Malik, H. J. (1986), "Order statistics from the linear- exponential distribution, Part II: Increasing hazard rate case”, Communications in Statistics - Theory and Methods, 15, 179-203.

Cordeiro, G., Ortega, E., and Popović, B. (2013), “The gamma-Lomax distribution”, J. Stat. Comput. Simul., 85(2), 305-319.

El-Bassiouny, A., Abdo, N., and Shahen, H. (2015), "Exponential Lomax distribution", Int. J. Comput. Appl., 121(13), 975-8887.

David, H. A. (1981 ), Order statistics, Second Edition, John Wiley \& Sons. New York. 
David, H. A. and Nagaraja, H. N. (2003), Order Statistics, third edition, John Wiley, New York.

Gradshteyn, I. S. and Ryzhik, I. M. (2014), Table of Integrals, Series, and Products, sixth edition, Academic Press, San Diego.

Joshi, P. C. (1978), "Recurrence relations between moments of order statistics from exponential and truncated exponential distributions", Sankhya, Series B, 39, 362-371.

Kamps, U. (1991), "A general recurrence relations for moments of order statistics in a class of probability distributions and characterizations". Metrika, 38, 215-225.

Kumar, D., Dey, S., and Nadarajah, S. (2016), "Extended exponential distribution based on order statistics", Communications in Statistics - Theory and Methods, 46, 9166-9184.

Kumar, D. and Dey, S. (2017), "Power generalized Weibull distribution based on order statistics", Journal of Statistical Research, 51, 61-78.

Kumar, D. (2017), “The Singh-Maddala distribution: Properties and Estimation”, International Journal of System Assurance Engineering and Management, 8, 1297-1311.

Lee, E. and Wang, J. (2003), "Statistical methods for survival data analysis, third edition", Wiley, New York.

Lemonte, A. and Cordeiro, G. (2013), “An extended Lomax distribution”, Statistics, 47, 800-816

Lin, G. D. (1988), "Characterizations of distributions via relations between two moments of order statistics”, Journal of Statistical Planning and Inference, 19, 73-80.

Malik, H. J., Balakrishnan, N., and Ahmed, S. E. (1988), "Recurrence relations and identities for moments of order statistics I: Arbitrary continuous distribution", Communications in Statistics - Theory and Methods, 17, 2623-2655.

Mohie El-Din, M. M., Mahmoudyuk, M. A. W., and Abu-Youssef, S. E. (1991), "Moments of order statistics from parabolic and skewed distributions and characterization of Weibull distribution", Communications in Statistics - Simulation and Computation, 20, 639-645.

Rady, E. A., Hassanein, W. A. and Elhaddad, T. A. (2016), "The power Lomax distribution with an application to bladder cancer data", Springer Plus, 5, 18-38.

Tahir, M., Cordeiroz, G., Mansoorx, M. and Zubair M. (2015), “The Weibull-Lomax distribution: Properties and applications”, textitHacet. J. Math. Stat., 44(2), 461-480.

Lee, E. T. and Wang, J. W. (2003), Statistical Methods for Survival Data Analysis, 3rd edition, Wiley, New York.

Received: October 31, 2017

Accepted: May 22, 2018 


\section{A Appendix}

Table A1: Mean of order statistics for different values of $\alpha, \beta$ and $\lambda$

\begin{tabular}{|c|c|c|c|c|c|c|c|}
\hline \multirow[b]{2}{*}{$n$} & \multirow[b]{2}{*}{$r$} & \multicolumn{3}{|c|}{$\beta=5, \lambda=1$} & \multicolumn{3}{|c|}{$\beta=10, \lambda=2$} \\
\hline & & $\alpha=0.5$ & $\alpha=1.5$ & $\alpha=3$ & $\alpha=0.5$ & $\alpha=1.5$ & $\alpha=3$ \\
\hline 1 & 1 & 1.5497 & 0.9298 & 0.7697 & 1.2760 & 1.0208 & 0.9316 \\
\hline \multirow[t]{2}{*}{2} & 1 & 1.0689 & 0.7697 & 0.6551 & 1.0896 & 0.9316 & 0.8605 \\
\hline & 2 & 2.0304 & 1.0899 & 0.8842 & 1.4624 & 1.1100 & 1.0027 \\
\hline \multirow[t]{3}{*}{3} & 1 & 0.9298 & 0.6990 & 0.5998 & 1.0208 & 0.8885 & 0.8236 \\
\hline & 2 & 1.3472 & 0.9109 & 0.7658 & 1.2272 & 1.0178 & 0.9342 \\
\hline & 3 & 2.3720 & 1.1795 & 0.9434 & 1.5801 & 1.1562 & 1.0370 \\
\hline \multirow[t]{4}{*}{4} & 1 & 0.8552 & 0.6551 & 0.5643 & 0.9806 & 0.8605 & 0.7990 \\
\hline & 2 & 1.1538 & 0.8307 & 0.7063 & 1.1413 & 0.9726 & 0.8975 \\
\hline & 3 & 1.5407 & 0.9912 & 0.8251 & 1.3129 & 1.0629 & 0.9709 \\
\hline & 4 & 2.6491 & 1.2423 & 0.9828 & 1.6691 & 1.1872 & 1.0590 \\
\hline
\end{tabular}

Table A2: Variance of order statistics for different values of $\alpha, \beta$ and $\lambda$

\begin{tabular}{|c|c|c|c|c|c|c|c|}
\hline \multirow[b]{2}{*}{$n$} & \multirow[b]{2}{*}{$r$} & \multicolumn{3}{|c|}{$\beta=5, \lambda=1$} & \multicolumn{3}{|c|}{$\beta=10, \lambda=2$} \\
\hline & & $\alpha=0.5$ & $\alpha=1.5$ & $\alpha=3$ & $\alpha=0.5$ & $\alpha=1.5$ & $\alpha=3$ \\
\hline 1 & 1 & 2.3608 & 0.0879 & 0.0419 & 0.1519 & 0.0259 & 0.0162 \\
\hline \multirow[t]{2}{*}{2} & 1 & 0.1786 & 0.0419 & 0.0259 & 0.0407 & 0.0162 & 0.0121 \\
\hline & 2 & 4.0807 & 0.0826 & 0.0316 & 0.1936 & 0.0198 & 0.0102 \\
\hline \multirow[t]{3}{*}{3} & 1 & 0.0879 & 0.0310 & 0.0207 & 0.0259 & 0.0135 & 0.0106 \\
\hline & 2 & 0.2439 & 0.0336 & 0.0180 & 0.0416 & 0.0105 & 0.0069 \\
\hline & 3 & 5.6489 & 0.0831 & 0.0278 & 0.2280 & 0.0182 & 0.0083 \\
\hline \multirow[t]{4}{*}{4} & 1 & 0.0615 & 0.0259 & 0.0179 & 0.0206 & 0.0121 & 0.0098 \\
\hline & 2 & 0.1003 & 0.0232 & 0.0139 & 0.0226 & 0.0081 & 0.0058 \\
\hline & 3 & 0.3127 & 0.0309 & 0.0150 & 0.0459 & 0.0088 & 0.0052 \\
\hline & 4 & 7.1206 & 0.0848 & 0.0259 & 0.2571 & 0.0175 & 0.0073 \\
\hline
\end{tabular}


Table A3: Skewness of order statistics for different values of $\alpha, \beta$ and $\lambda$

\begin{tabular}{|c|c|c|c|c|c|c|c|}
\hline \multirow[b]{2}{*}{$n$} & \multirow[b]{2}{*}{$r$} & \multicolumn{3}{|c|}{$\beta=5, \lambda=1$} & \multicolumn{3}{|c|}{$\beta=10, \lambda=2$} \\
\hline & & $\alpha=0.5$ & $\alpha=1.5$ & $\alpha=3$ & $\alpha=0.5$ & $\alpha=1.5$ & $\alpha=3$ \\
\hline 1 & 1 & 30.658 & 1.1818 & 0.0768 & 11.7334 & 0.1025 & 0.0437 \\
\hline \multirow[t]{2}{*}{2} & 1 & 6.1766 & 0.0768 & 0.0002 & 0.8776 & 0.0437 & 0.1897 \\
\hline & 2 & 31.1178 & 2.1342 & 0.2609 & 13.4439 & 0.5748 & 0.0183 \\
\hline \multirow[t]{3}{*}{3} & 1 & 1.1818 & 0.0059 & 0.0097 & 0.1025 & 0.1304 & 0.2547 \\
\hline & 2 & 8.9202 & 0.2536 & 0.0145 & 1.9846 & 0.0172 & 0.0319 \\
\hline & 3 & 30.8511 & 2.6949 & 0.4273 & 13.5957 & 0.9108 & 0.1003 \\
\hline \multirow[t]{4}{*}{4} & 1 & 0.4034 & 0.0002 & 0.0193 & 0.0020 & 0.1897 & 0.2913 \\
\hline & 2 & 2.3007 & 0.0597 & 0.00 & 0.6335 & 0.0054 & 0.0786 \\
\hline & 3 & 9.8308 & 0.4111 & 0.0486 & 2.4017 & 0.0934 & 0.0010 \\
\hline & 4 & 30.5981 & 3.0554 & 0.5605 & 13.5387 & 1.1386 & 0.1894 \\
\hline
\end{tabular}

Table A4: Kurtosis of order statistics for different values of $\alpha, \beta$ and $\lambda$

\begin{tabular}{rrrrrrrrrr}
\hline & \multicolumn{3}{c}{$\beta=5, \lambda=1$} & & \multicolumn{3}{c}{$\beta=10, \lambda=2$} \\
\cline { 2 - 6 } \cline { 7 - 9 }$n$ & $r$ & $\alpha=0.5$ & $\alpha=1.5$ & $\alpha=3$ & & $\alpha=0.5$ & $\alpha=1.5$ & $\alpha=3$ \\
\hline 1 & 1 & 14.7921 & 7.16626 & 3.4837 & & 48.2997 & 4.1793 & 3.4336 \\
2 & 1 & 29.5560 & 3.4837 & 3.0095 & & 6.5101 & 3.4336 & 3.4209 \\
& 2 & 14.8156 & 9.2913 & 3.9993 & & 51.7441 & 5.0312 & 3.5012 \\
3 & 1 & 7.16625 & 3.1178 & 2.9378 & & 4.1793 & 3.3855 & 3.4513 \\
& 2 & 37.1271 & 3.9743 & 3.2038 & & 8.1988 & 3.4671 & 3.3094 \\
& 3 & 14.6321 & 10.4404 & 4.3370 & & 51.7369 & 5.5669 & 3.6625 \\
4 & 1 & 4.6305 & 3.0095 & 2.9151 & & 3.6337 & 3.4209 & 3.4894 \\
& 2 & 9.4917 & 3.3785 & 3.0952 & & 5.0530 & 3.2772 & 3.2661 \\
& 3 & 39.2805 & 4.2729 & 3.3013 & & 8.7362 & 3.6075 & 3.2771 \\
& 4 & 14.4867 & 11.1683 & 4.5789 & & 51.3831 & 5.9166 & 3.6969 \\
\hline
\end{tabular}

